\title{
Caracteristiques de morphologie générale et de croissance du Cyprinidae africain Labeobarbus batesii dans la plaine inondable des Mbô, Cameroun
}

\author{
Claudine TEKOUNEGNING TIOGUE ${ }^{1^{*}}$, Minette Tabi EYANGO TOMEDI ${ }^{2}$, \\ David NGUENGA $^{3}$ et Joseph TCHOUMBOUE ${ }^{4}$
}

\author{
IUniversité de Dschang, Faculté d'Agronomie et des Sciences Agricoles (FASA), Laboratoire d'Ichtyologie et \\ d'Hydrobiologie Appliquée, BP 222 Dschang Cameroun. \\ ${ }^{2}$ Université de Douala, Institut des Sciences Halieutiques de Yabassi (ISH), BP 2701 Douala, Cameroun. \\ ${ }^{3}$ Institut de Recherche Agricole pour le Développement, Unité de Recherche Piscicole, BP 255 Foumban, \\ Cameroun. \\ ${ }^{4}$ Université des Montagnes, Bangangté, Cameroun. E-mail : jtchoumboue@yahoo.fr \\ "Auteur correspondant ; E-mail : tekou_claudine@yahoo.fr; Tél : + 23775079565
}

\section{RESUME}

Des investigations de quelques aspects de la biologie et de l'écologie du poisson d'eau douce Labeobarbus batesii dans la plaine inondable des Mbô au Cameroun, ont été menées entre mai 2008 et octobre 2009. L'étude révèle que cette espèce est abondante et répandue dans les cours d'eaux de cette plaine. L'abondance en nombre a été importante pendant la saison pluvieuse et chez les poissons de taille comprise entre 23 et $24 \mathrm{~cm}$. En général cette espèce a une croissance isométrique (sans distinction de sexe); atteignant une longueur et poids totaux maximum de $50,5 \mathrm{~cm}$ et $1300 \mathrm{~g}$ respectivement. Toutefois, cette croissance a présentée des déviations saisonnières et annuelles, c'est ainsi qu'en saison des pluies, elle passe d'allométrie positive au cours de l'année 2008 à l'allométrie négative en 2009. Le facteur de condition K a varié entre 0,897 à 1,096 (moyenne $=1,028 \pm 0,118$ ); ces valeurs n'ont pas varié avec le sexe, ni entre les tailles. Au vu de ses caractéristiques morphologiques et de croissance, cette espèce pourrait devenir un candidat potentiel pour la domestication afin d'améliorer l'alimentation humaine. Toutefois des investigations sur les caractéristiques reproductives et les habitudes alimentaires de cette espèce pourront compléter ce travail.

(C) 2010 International Formulae Group. All rights reserved.

Mots clés : Carpe africaine, fréquence de distribution, croissance, plaine inondable, Cameroun.

\section{INTRODUCTION}

Dans de nombreuses régions du monde, les Cyprinidae constituent un modèle de base de l'aquaculture. En Afrique, Cyprinus carpio, importée d'Eurasie est la principale espèce exotique produite (Lazard, 2007). Selon Lazard et Levêque (2009), les espèces exotiques introduites dans ce continent n'ont pas atteint leur but de stimuler le développement d'une filière de production aquacole ; cela est en grande partie lié au fait que le choix de l'espèce ne constitue pas le principal frein au développement de ce secteur sur ce continent. Globalement, l'Afrique abrite une biodiversité d'espèces de poissons d'eau douce largement suffisante pour soutenir un développement aquacole tout à fait comparable à celle qu'abrite l'Asie (3200 
et 3000 respectivement). Mais contrairement à l'Asie, plus de $90 \%$ des pisciculteurs africains sont des opérateurs artisans sans véritable technicité, d'où la nécessité de disposer d'espèces d'élevage facile à gérer (Lazard et Levêque, 2009). Les mêmes auteurs rappellent que l'utilisation d'espèces autochtones ou naturalisées constitue dans ce contexte un avantage comparatif sur des espèces exotiques, notamment pour l'approvisionnement en géniteurs et en alevins. Aucun Cyprinidae africain parmi près de 526 espèces décrites (Stiassny et al., 2007) n'est actuellement utilisé en aquaculture. De ces espèces décrites, 79 sont primaires en basse Guinée. Le genre Labeobarbus est plus répandu et s'y retrouve dans la majorité des cours d'eaux continentaux. Il pourrait jouer le même rôle que la carpe commune en aquaculture. $\mathrm{Au}$ Cameroun, la plaine inondable des Mbô est alimentée par de nombreux cours d'eaux importants dont les ressources piscicoles jouent un rôle significatif dans l'économie et l'alimentation de la population locale. Dans cette zone, la population a toujours consommé les poissons d'eau douce locaux et cette consommation est dix fois supérieure à celle du centre urbain de Yaoundé (Pouomogne, 2008); Clarias gariepinus y est le candidat préféré pour la pisciculture tandis que Clarias jaensis et Labeobarbus batesii sont favorables dans les plats traditionnels et de mariages, ainsi que dans d'autres célébrations coutumières (Pouomogne, 2008). Cette zone joue ainsi un important rôle comme réserve naturelle pour ces espèces. Ces deux dernières espèces peuvent aussi bien devenir des candidats potentiels pour la pisciculture. Cependant, si très peu de données de littérature existent sur la biologie des populations naturelles des Clariidae de cette zone, rien n'est connu de celles des Cyprinidae. L'objectif de ce travail est donc d'étudier la biologie et l'écologie des populations du Labeobarbus batesii dans la plaine inondable des Mbô, en vue de leur domestication. Ce travail fournit des informations sur la longueur, le poids, les fréquences de distribution mensuelle et par taille, les paramètres des relations longueurlongueur, poids-poids, longueur-poids, et le facteur de condition $\mathrm{K}$ de Labeobarbus batesii des cours d'eaux de la plaine d'inondation des Mbô.

\section{MATERIEL ET METHODES \\ Zone de l'étude et sites de collecte des poissons}

La plaine inondable des Mbô (5 $5^{\circ} 10^{\prime}$ $5^{\circ} 30^{\prime} \mathrm{N}, 9^{\circ} 50^{\prime}-10^{\circ} 10^{\prime} \mathrm{E}$; altitude : $700 \mathrm{~m}$ ) couvre trois arrondissements : Santchou dans le département de la Menoua et Kekem dans le département du Haut-Nkam, tous situés dans la Région de l'Ouest Cameroun; et Melong dans le département du Mungo, région du Littoral au Cameroun en Afrique Centrale.

Le climat chaud et humide est de type camerounien à régime des pluies pseudo tropical avec une moyenne de précipitations de $1860 \mathrm{~mm}$. La plaine des Mbô est caractérisée par deux saisons: une saison sèche allant de mi - novembre à mi - mars, au cours de laquelle les températures varient de $18{ }^{\circ} \mathrm{C}$ à $30^{\circ} \mathrm{C}$; et une saison de pluie de mi mars à mi - novembre avec des températures de $17{ }^{\circ} \mathrm{C}$ à $26^{\circ} \mathrm{C}$. L'humidité relative varie de 49,0 à 97,9\% entre les deux saisons.

La plaine des Mbô est parcourue par des cours d'eaux à trois origines : Les eaux qui naissent au pied du mont Bamboutos s'unissent à Dschang pour former la rivière Menoua qui dans la plaine rejoint le petit Nkam formé par les eaux issues du massif de Manengouba. Le cours d'eau ainsi formé va rencontrer la rivière «Black water» et les eaux issues du massif de Bana pour former le grand Nkam au niveau de Melong qui se jette dans l'océan atlantique à travers le fleuve Wouri (Olivry, 1986) (Figure 1). Le modelé de la plaine dépend des crues de ces cours d'eaux. Selon la fréquence et l'intensité des pluies, ces eaux submergent le plus souvent les berges en saison des pluies pour inonder les bas fonds environnants. Le nombre de crues varie d'une année à l'autre. La relative abondance des poissons se caractérise toutefois par une saisonnalité marquée de la 


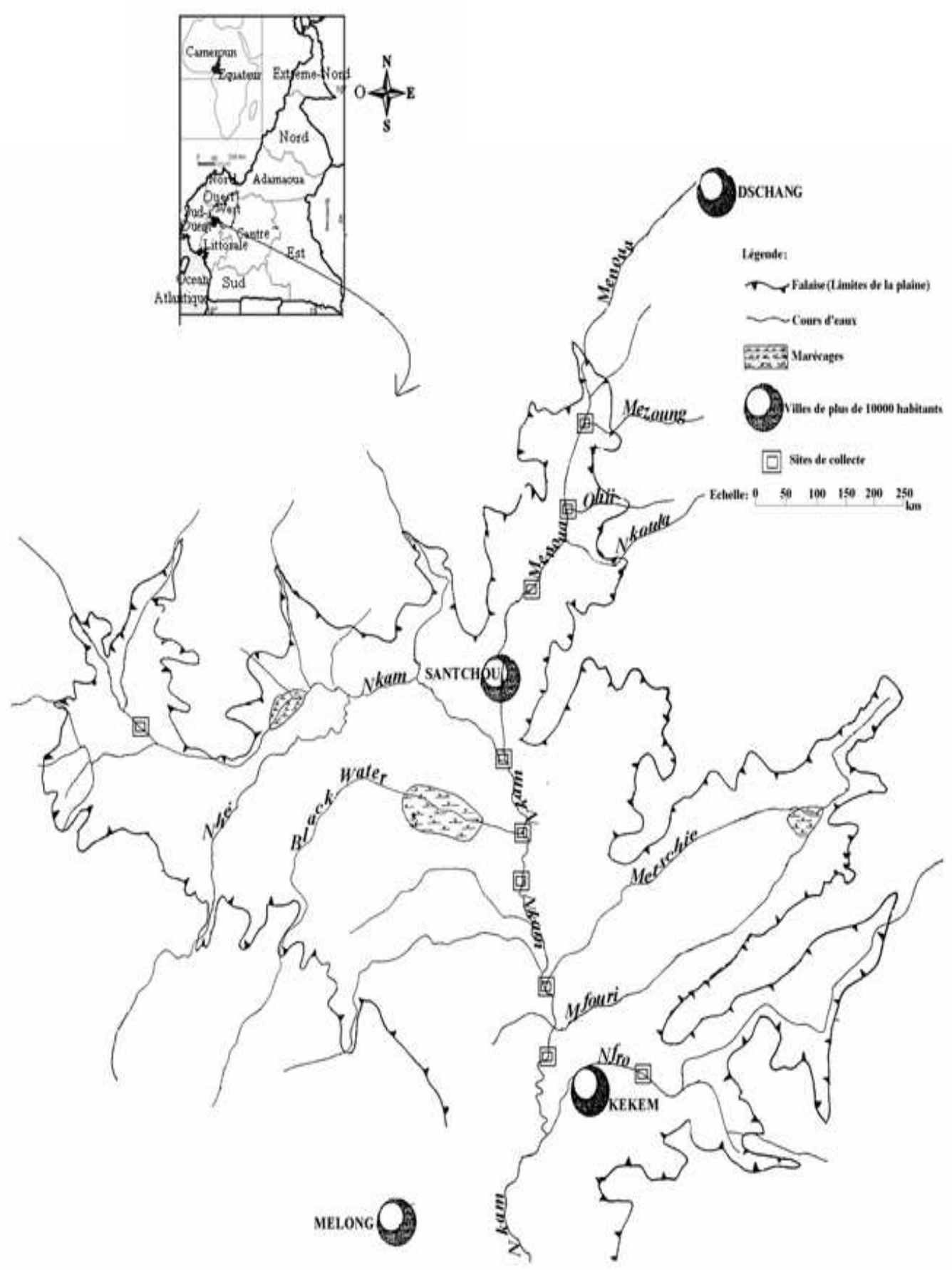

Figure 1 : Plaine des Mbô : réseau hydrographique et sites de collecte des poissons. Source Olivry, 1986. 
pêche en fonction de la crue, de la décrue et des migrations des poissons qui sont associées.

Les dix sites de collectes des poissons ont été choisis le long des rivières Menoua, petit Nkam, et grand Nkam : cinq aux points de confluence de deux cours d'eaux et cinq autres entre deux zones de confluence des cours d'eaux.

\section{Collecte des données}

Les poissons ont été collectés mensuellement entre Mai 2008 et Octobre 2009 par des méthodes artisanales de pêche (filets maillants de type «épervier», des hameçons et des nasses appâtées). Ils ont été transportés au laboratoire dans une glacière, et ont été identifiés au niveau des familles et des espèces par la clé d'identification de Stiassny et al. (2007). Les mensurations ont été effectuées sur un échantillon de 486 poissons. Des mesures de longueur ont été faites à l'aide d'un ichtyomètre à $0,1 \mathrm{~cm}$ près de l'extrémité antérieure du museau jusqu'à la base de la nageoire caudale pour la longueur standard (LS), et jusqu'à sa pointe pour la longueur totale (LT). Le poids total (PT) de chaque poisson a été mesuré à l'aide d'une balance électronique (OHAUS DIAL-OGRAM) de précision 0,1g. Après dissection et examen à l'œil nu des gonades, le sexe de chaque poisson a été déterminé ; les poissons immatures et ceux conservés dans du formol pour l'identification ultérieure de l'espèce ont été considérés comme étant à sexe indéterminé. Le poids éviscéré (Pé) a été obtenu après extraction des viscères, à l'aide de la précédente balance.

Les relations longueur totale-longueur standard et poids total-poids éviscéré ont été établies par régression linéaire selon la méthode des moindres carrés, et les équations ont été les suivantes : $\mathrm{LT}=\mathrm{a}+\mathrm{b} \mathrm{LS}$ et $\mathrm{Pt}=\mathrm{a}$ $+\mathrm{b}$ Pé $(\mathrm{a}=$ ordonné à l'origine et $\mathrm{b}=$ pente de la droite de régression)

La relation longueur-poids a été établie sous la forme $\mathrm{PT}=\mathrm{a} \mathrm{LT}^{\mathrm{b}}$ (Le Cren, 1951) avec a comme constante de la régression, b le coefficient de régression, $\mathrm{LT}=$ longueur totale $(\mathrm{cm})$ et $\mathrm{PT}=$ poids total $(\mathrm{g})$. Des relations par régression linéaire ont été établies entre la longueur standard (LS) et la longueur totale (LT), et entre le poids total (PT) et le poids éviscéré (Pé).

En supposant que b est souvent proche de 3, le facteur de condition $\mathrm{K}$ a été calculé suivant Ricker (1975) : K= $100 \mathrm{X} \mathrm{PT/} \mathrm{LT}^{3}$ en fonction du mois, du sexe et de la taille. Dans ce dernier cas, les poissons ont été regroupés en fonction de la longueur totale par classe de $5 \mathrm{~cm}$ d'intervalle, soit au total 7 classes.

\section{Analyses statistiques}

Les moyennes ont été comparées avec le test ANOVA I suivi du test de Duncan; la signification statistique du coefficient de détermination $r^{2}$ a été estimée et le test $t$ de student a été utilisé pour vérifier si les valeurs de $\mathrm{b}$ étaient significativement différentes de la valeur isométrique $\mathrm{b}=3$ au seuil de $5 \%$. Toutes ces analyses ont été effectuées au moyen du logiciel SPSS version 12.0. Les figures ont été obtenues grâce à Microsoft Office Excel 2003.

\section{RESULTATS}

\section{Caractéristiques morphométriques et fréquences de distribution}

Les longueurs totales de 486 échantillons de Labeobarbus batesii collectés dans la plaine des Mbô varient de 17 à 50,5 $\mathrm{cm}$ avec les valeurs correspondantes de longueur standard de 15,5 à 41,5 cm. Les poids totaux varient de 40 à $1300 \mathrm{~g}$, avec les valeurs correspondantes de poids éviscéré de 35 à $1225 \mathrm{~g}$. Les fréquences de distribution mensuelle et de longueur totale de la population de cette espèce sont présentées dans les Figures $2 \mathrm{a}$ et $2 \mathrm{~b}$; la distribution mensuelle est tri modale: le mode le plus élevé se situe en septembre 2008, le moyen en octobre 2009 et le plus faible en mai 2009. La distribution en fonction de la longueur totale a une allure gaussienne, la taille de la population est concentrée entre 19 et $31 \mathrm{~cm}$, la classe la plus abondante étant [23-24] cm. 


\section{Relation longueur-longueur}

La relation longueur totale-longueur standard chez Labeobarbus batesii de la plaine inondable des Mbô a été hautement significative $(\mathrm{P}<$ 0,0001). L'équation de régression a été : $\mathrm{LT}=1,163 \mathrm{LS}+1,3337\left(\mathrm{r}^{2}\right.$ $=0,9753, \mathrm{~N}=486)$, (Figure 3a).

\section{Relation poids-poids}

La relation poids total-poids éviscéré de Labeobarbus batesii de la plaine inondable des Mbô a été hautement significative $(\mathrm{P}<$ 0,001). L'équation de régression a été : $\mathrm{PT}=$ 1,1201 Pé + 9,6869 $\left(\mathrm{r}^{2}=0,9879, \mathrm{~N}=426\right)$, (Figure 3b).

\section{Relation longueur-poids}

La relation longueur totale- poids total de Labeobarbus batesii de la plaine inondable des Mbô a été hautement significative $(\mathrm{P}<$ 0,0001), l'équation générale a été de PT = $0,0101 \mathrm{LT}^{3,0033}$ et $\mathrm{r}^{2}=0,941$ (Figure 4). Les paramètres mensuels de cette relation sont présentés dans le Tableau 1: La croissance varie d'allométrie positive $(\mathrm{b}>3$ ) (de mai 2008 à septembre 2008) à l' allométrie négative $(b<3)$ (de novembre 2008 à octobre 2009). Toutefois, les valeurs isométriques de b $(b=3)$ ont été enregistrées en août, octobre et décembre 2008, en mars et mai 2009. La valeur générale de b a été comparable à $3(\mathrm{P}>$ 0,05). En considérant le sexe, les équations de la droite ont été de PT $=0,0124 \mathrm{LT}^{2,9453}\left(\mathrm{r}^{2}=\right.$ 0,9483, $\mathrm{N}=254$ ) chez les femelles, de PT= $0,0131 \mathrm{LT}^{2,9256}\left(\mathrm{r}^{2}=0,9221, \mathrm{~N}=172\right)$ chez les mâles, et PT $=0,0126 \mathrm{LT}^{2,9276}\left(\mathrm{r}^{2}=0,8119, \mathrm{~N}\right.$ $=60$ ) chez les poissons à sexe indéterminé (Figure 4). Aucune différence significative n'a été observée entre les sexes pour le coefficient b $(p>0,05)$ et quelque soit le sexe, ce coefficient a été comparable à la valeur isométrique. Les coefficients de détermination ont été hautement significative $(\mathrm{P}<0,001)$.

\section{Facteur de condition}

Les valeurs mensuelles du facteur de condition sont présentées dans le Tableau 1 ; elles varient de 0,897 à 1,096 (moyenne = $1,028 \pm 0,118$ ). Les Figures 5 a et $5 b$ illustrent les variations de $\mathrm{K}$ en fonction du sexe et de la classe de taille. Le facteur de condition a varié de 1,064 à 1,147 (moyenne $=1,0126 \pm 0,071$ ) chez les femelles, de 0,825 à 1,185 (moyenne $=1,004 \pm 0,022$ ) chez les mâles et de 0,860 à 1,072 (moyenne $=1,0415 \pm 0,0305$ ) chez les individus à sexe indéterminé. Toutefois, aucune différence significative n'a existée entre les sexes pour ce paramètre $(\mathrm{P}<0,01)$. Les valeurs les plus élevées ont été enregistrées en saison de pluies (mai, août, septembre, octobre et novembre) chez les mâles en 2008 et en septembre et octobre 2009 chez les femelles. Le facteur de condition a été > 1 quelque soit la taille et a varié de 1,009 pour les poissons de taille comprise entre 25 et $30 \mathrm{~cm}, 45$ et $55 \mathrm{~cm}$ et à 1,084 pour ceux de taille comprise entre 40 et $45 \mathrm{~cm}$ de longueur totale. Ces différences n'ont pas été significatives $(\mathrm{P}>0,05)$.

\section{DISCUSSION}

La taille maximale observée chez Labeobarbus batesii dans la plaine des Mbô a été de $505 \mathrm{~mm}$ LT chez une femelle, correspondant à un poids de 1300 g. Cette espèce appartient au genre Labeobarbus Rupell, 1836 qui comprend des espèces de tailles assez grandes à l'état adulte (Stiassny et al., 2007). Elle pourrait atteindre des tailles plus grandes car il y existe un dimorphisme sexuel, les mâles étant généralement plus développés que les femelles. Selon Fontaine et al. (2009), cette caractéristique biologique est un bon indicateur des performances zootechniques susceptibles de s'exprimer en milieu d'élevage. Legendre et Albaret (1991) confirment d'ailleurs que la taille maximale d'un poisson constitue un indicateur de sa vitesse de croissance et que les plus grandes espèces sont très généralement celles qui possèdent le meilleur potentiel d'adaptation à différents milieux. 

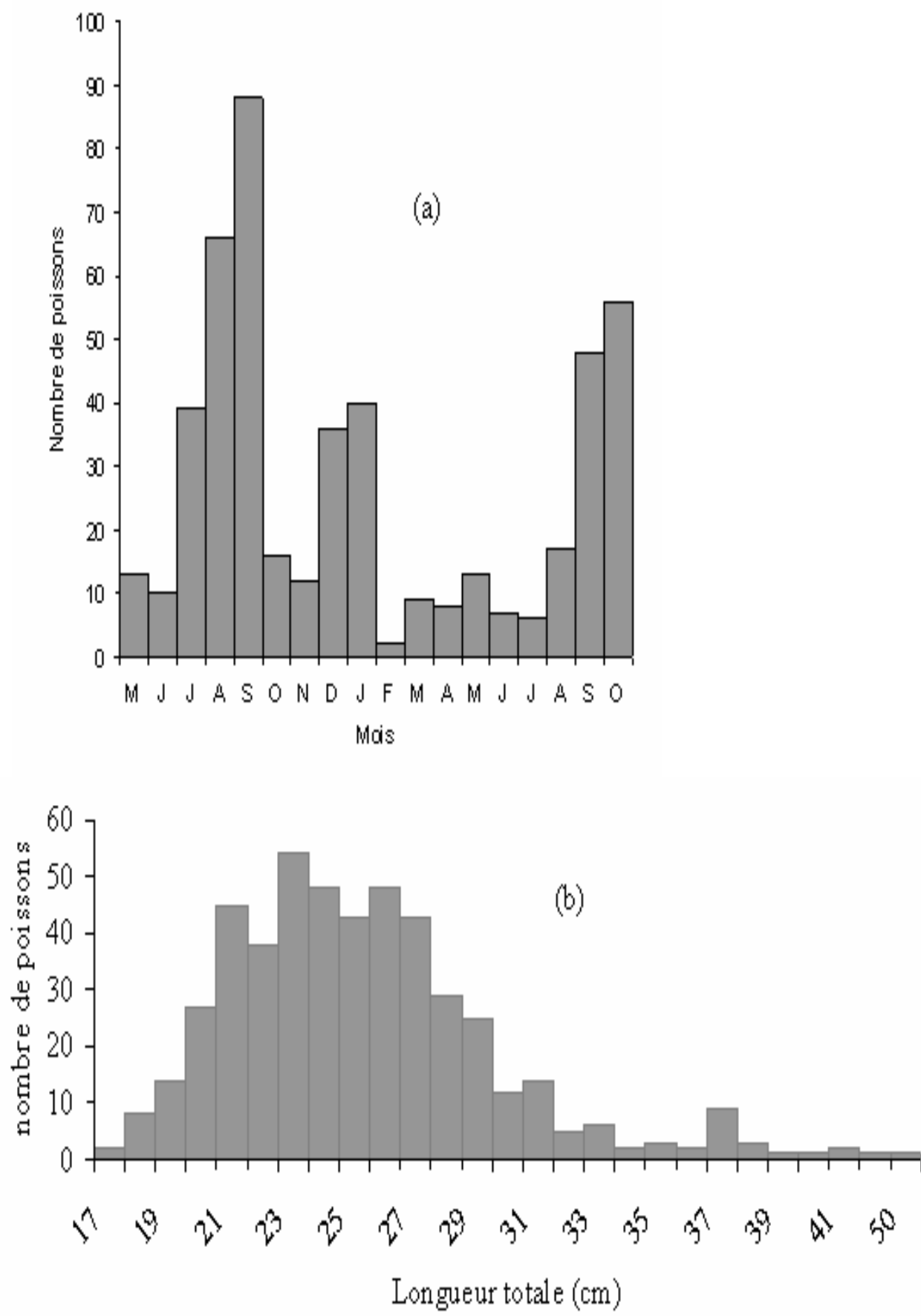

Figure 2 : Fréquence de distribution mensuelle (a) et par taille (b) de Labeobarbus batesii dans la plaine inondable des Mbô (mai 2008- octobre 2009). 

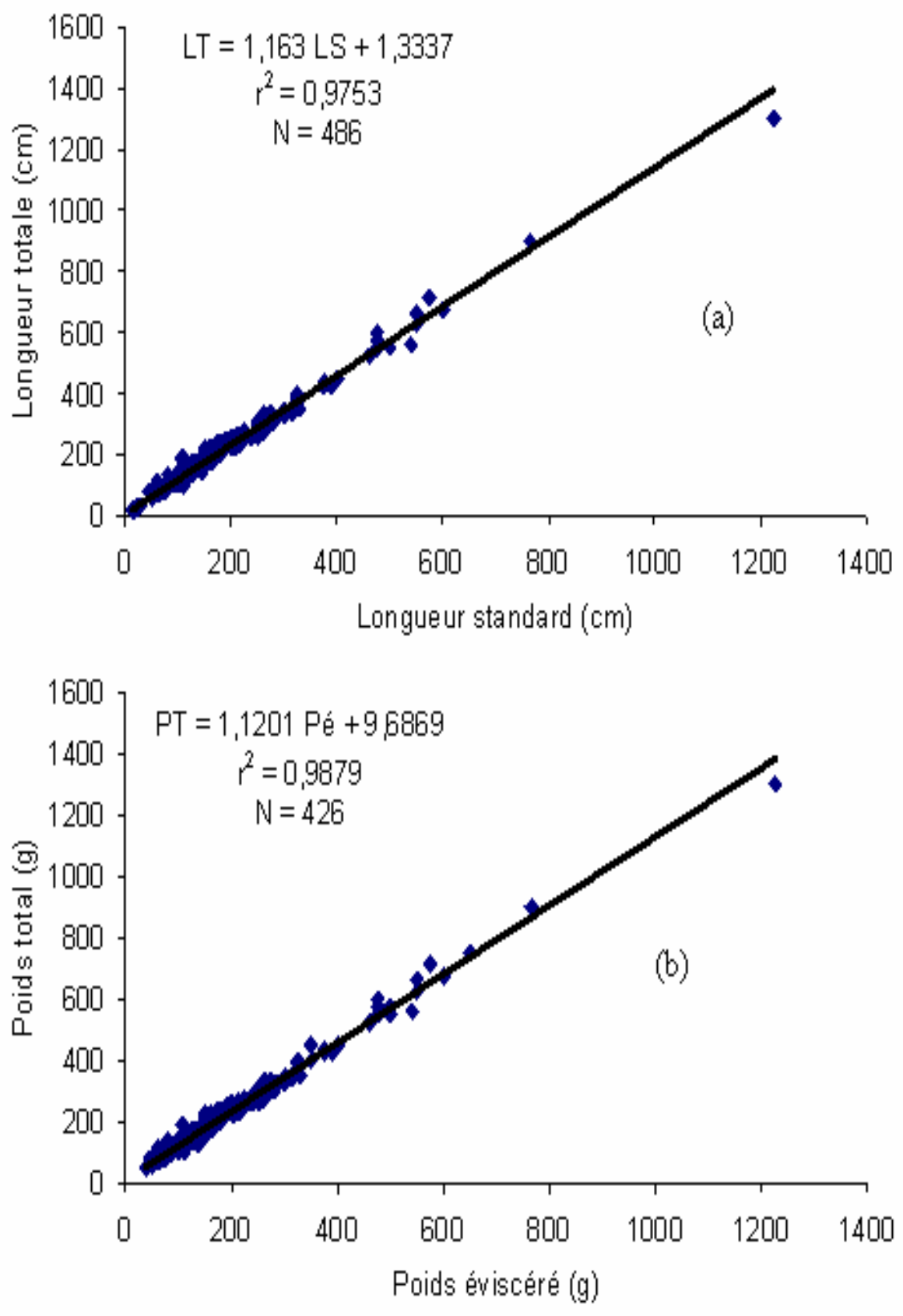

Figure 3 : Relations Longueur totale-Longueur standard (a), poids total- poids éviscéré (b) chez Labeobarbus batesii dans la plaine inondable des Mbô.

$\mathrm{LT}=$ longueur totale, $\mathrm{LS}=$ longueur standard, $\mathrm{PT}=$ Poids total, Pé = poids éviscéré, $\mathrm{r}^{2}=$ coefficient de détermination, $\mathrm{N}=$ nombre de poissons. 

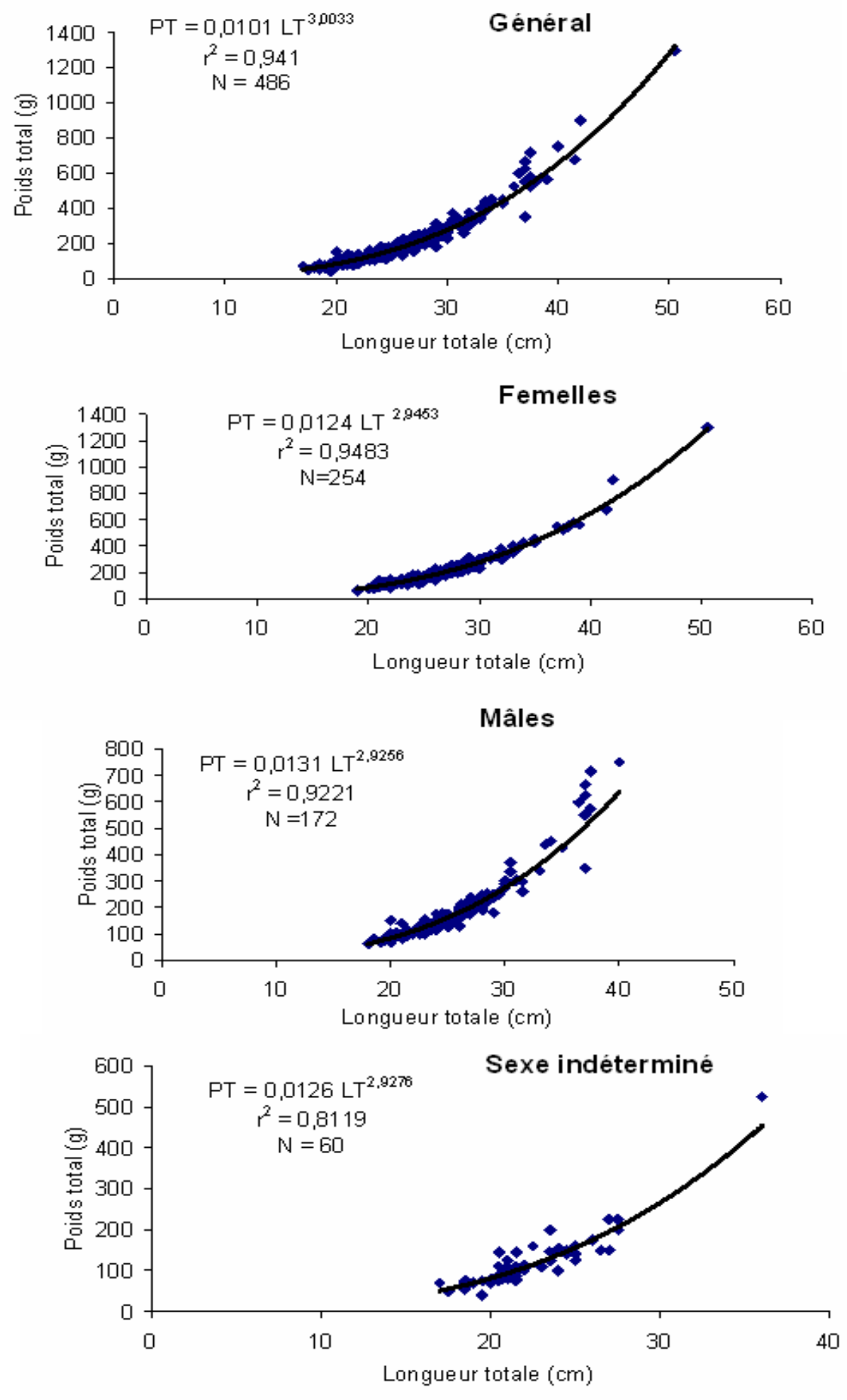

Figure 4 : Relation longueur-poids chez Labeobarbus batesii dans la plaine inondable des Mbô (mai 2008-octobre 2009).

$\mathrm{PT}=$ poids total, $\mathrm{LT}=$ longueur totale, $\mathrm{r}^{2}=$ coefficient de détermination, $\mathrm{N}=$ nombre de poissons. 

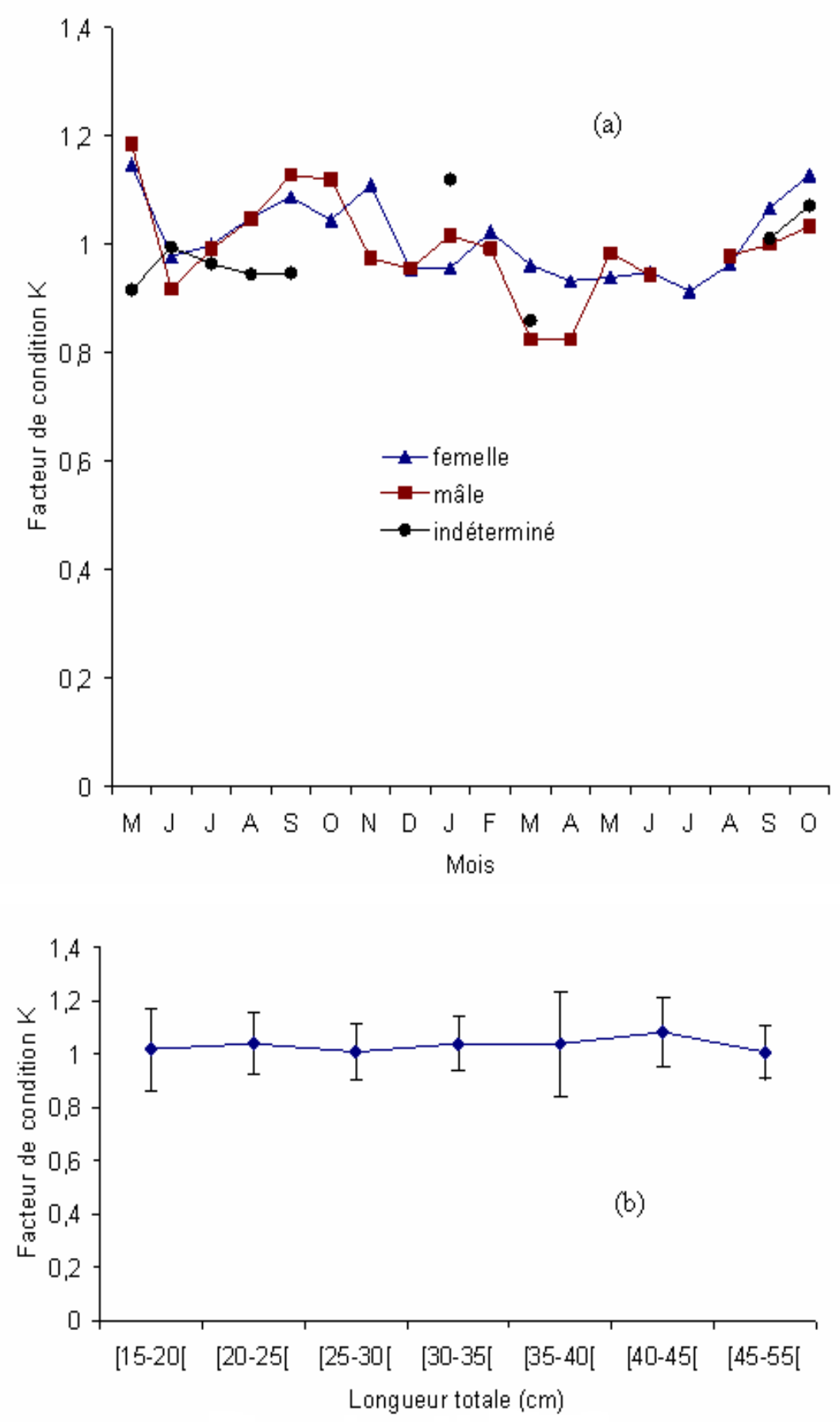

Figure 5 : Facteur de condition en fonction du sexe (a) et de la taille (b) chez Labeobarbus batesii dans la plaine inondable des Mbô (mai 2008-actobre 2009). 
Tableau 1 : Paramètres mensuels de la relation poids - longueur et facteur de condition $\mathrm{K}$ chez Labeobarbus batesii dans la plaine d'inondation des Mbô (Mai 2008-Octobre 2009).

\begin{tabular}{llllllc}
\hline Mois & $\mathbf{N}$ & $\mathbf{r}^{\mathbf{2}}$ & $\mathbf{a}$ & $\mathbf{b}$ & Type de croissance & $\mathbf{K}$ \\
\hline Mai 2008 & 13 & 0,90 & 0,0015 & 3,622 & Allométrique positive & $1,088 \pm 0,191$ \\
Juin & 10 & 0,93 & 0,0022 & 3,453 & Allométrique positive & $0,955 \pm 0,108$ \\
Juillet & 35 & 0,97 & 0,0056 & 3,179 & Allométrique positive & $0,986 \pm 0,084$ \\
Août & 57 & 0,94 & 0,0097 & 3,019 & isométrique & $1,038 \pm 0,102$ \\
Septembre & 85 & 0,96 & 0,0063 & 3,167 & Allométrique positive & $1,096 \pm 0,131$ \\
Octobre & 46 & 0,90 & 0,013 & 2,935 & Isométrique & $1,086 \pm 0,151$ \\
Novembre & 25 & 0,924 & 0,0459 & 2,565 & Allométrique négative & $1,094 \pm 0,171$ \\
Décembre & 31 & 0,983 & 0,0073 & 3,084 & Isométrique & $0,960 \pm 0,078$ \\
Janvier 2009 & 34 & 0,941 & 0,0224 & 2,741 & Allométrique négative & $1,003 \pm 0,130$ \\
Février & 2 & 1 & 1,3517 & 0,875 & Allométrique négative & $1,008 \pm 0,016$ \\
Mars & 9 & 0,955 & 0,0117 & 2,919 & Isométrique & $0,897 \pm 1,16$ \\
Avril & 8 & 0,963 & 0,0145 & 2,862 & Allométrique négative & $0,906 \pm 0,090$ \\
Mai & 13 & 0,980 & 0,0104 & 2,969 & Isométrique & $0,949 \pm 0,085$ \\
Juin & 7 & 0,972 & 0,0535 & 2,472 & Allométrique négative & $0,971 \pm 0,100$ \\
Juillet & 5 & 0,946 & 0,0561 & 2,453 & Allométrique négative & $0,914 \pm 0,041$ \\
Août & 17 & 0,968 & 0,0051 & 3,197 & Allométrique positive & $0,969 \pm 0,073$ \\
Septembre & 43 & 0,922 & 0,0377 & 2,590 & Allométrique négative & $1,038 \pm 0,100$ \\
octobre & 46 & 0,926 & 0,0172 & 2,859 & Allométrique négative & $1,086 \pm 0,241$ \\
général & 444 & 0,943 & 0,0109 & 2,980 & Isométrique & $1,028 \pm 0,118$ \\
\hline N = nombre de poissons, $\mathrm{r}^{2}=$ coefficient de détermination, a et b = paramètres estimatifs de la relation longueur-poids, \\
K = facteur de condition K. & & & & &
\end{tabular}

Ces résultats préliminaires sur la biologie des populations de Labeobarbus batesii de la plaine des Mbô montrent une fluctuation mensuelle en abondance aussi bien en nombre qu'en taille des individus. La fréquence de distribution par taille a une allure gaussienne et montre que le plus grand nombre d'individus collectés est concentré entre 190 et $310 \mathrm{~mm} \mathrm{LT}$, soit 90,53\% de la population. Cela peut s'expliquer par le fait que les techniques de pêche utilisées ne capturent que des poissons de cette taille, ou alors que l'espérance de vie chez cette espèce soit très courte et les poissons meurent rapidement. La fréquence de distribution mensuelle montre un grand nombre d'individus collecté en pleine saison de pluies. Ces résultats suggèrent que la saison des pluies entraîne des modifications sur l'habitat, lesquelles sont en faveur de la croissance des poissons. Pendant les mois de saison sèche, la plupart des habitats s'assèchent et la collecte des échantillons devient difficile. Aussi les jeunes poissons inhabitués aux différentes techniques de pêches sont les plus capturés en saison pluvieuse où ils sortent de leurs niches, attirés par la richesse en substances nutritives des eaux de ruissellement qui inondent la plaine. Des résultats similaires ont été rapportés chez le Décapode Cragon orangon, chez les Potamonautidae Sudanonautes africana, Sudanonautes orthostylis, Potamonautes granularis et Sudanonautes floweri (Arimoro et Orogun, 2008).

Quoique plusieurs études soient faites sur la relation poids-longueur, peu de facteurs de conversion autorisent actuellement de calculer les longueurs totale et standard, les poids total et éviscéré à partir d'une mensuration connue (Montcho et al., 2009). Les relations longueur totale-longueur standard et poids total-poids éviscéré n'ont pas beaucoup d'intérêt biologique en ellesmêmes, mais elles permettent de pouvoir corriger les données manquantes et d'interpréter aisément les résultats exprimés 
en l'un ou l'autre des paramètres longueur ou poids (Chikou, 2006). Le même auteur rappelle que la longueur totale est souvent utilisée pour sa facilité et sa rapidité de mesure ; et convient mieux à la mesure d'un grand nombre de poissons. La longueur standard a l'avantage d'éviter les erreurs dues aux nageoires caudales abîmées accidentellement dans les pêches ou lors des combats intra ou interspécifiques et qui faussent la mesure de la longueur totale (Chikou, 2006). Elles permettent donc chez Labeobarbus batesii comme chez Lates niloticus (Montcho et al., 2009), de déterminer dans un premier temps le facteur de conversion pour la longueur totale- longueur standard et pour le poids total- poids éviscéré, et dans un deuxième temps, de servir comme la base de la comparaison pour les études basées sur ces mensurations. Ainsi les longueurs totale et standard sont fortement corrélées chez Labeobarbus batesii $\left(\mathrm{r}^{2}=\right.$ 0,975) dans la plaine inondable des Mbô comme chez six espèces de Clariidae $\left(r^{2}=\right.$ 0,962) dans le delta de l'Ouémé (Chikou, 2006) et chez Lates niloticus $\left(\mathrm{r}^{2}=0,99\right)$ dans la rivière pendjari au Bénin (Montcho et al., 2009) . Le poids éviscéré est le plus souvent préféré au poids total afin de minimiser les erreurs liées à l'état de réplétion de l'estomac (Levêque et Paugy, 1999) dans la détermination du facteur de condition et $\mathrm{du}$ rapport gonadosomatique. Il a été fortement corrélé au poids total chez Labeobarbus batesii $\left(\mathrm{r}^{2}=0,987\right)$ dans la plaine des Mbô ; ces résultats sont comparés à ceux rapportés par Chikou (2006) dans le delta de l'Ouémé chez les Clariidae.

La longueur totale est fortement corrélée $\left(\mathrm{r}^{2}=0,944\right)$ au poids corporel chez cette espèce. Ces résultats sont similaires à ceux rapportés chez Labeo coubie $\left(\mathrm{r}^{2}=0,90\right)$ et Labeo senegalensis $\left(r^{2}=0,95\right)$ dans la rivière Cross au Nigeria (Offem et al., 2009). La valeur du coefficient de régression $b$ donne des informations sur le type de croissance du poisson (Montcho et al., 2009); celles de Labeobarbus batesii de la Plaine des Mbô ont varié dans l'ensemble de 2,453 à 3,622 (si on ne tient pas compte du mois de février où la croissance a été sensiblement linéaire (avec deux spécimens) et $\mathrm{b}$ a été de 0,875). Cette fourchette est concordante avec celle habituellement rapportée et admise par la littérature et qui situerait cette valeur entre 2,50 et 3,50 (Pauly et Gayanilo, 1997) ou entre 2,50 et 4,00 (Offem et al., 2009), ou entre 2,00 et 4,00 (Montchowui et al., 2009). $\mathrm{Au}$ cours de certains mois, la valeur de b a été de 3, ce qui signifie que la forme du corps n'a pas changé avec la croissance qui est ainsi dite isométrique. Des déviations de cette croissance isométrique sont observées chez cette espèce et la forme de son corps a changé au cours de sa croissance (Coulibaly, 2008). C'est ainsi que de Mai 2008 à septembre 2008, les valeurs de b ont été significativement > 3 montrant ainsi que la croissance est en faveur du poids, donc est allométrique positive. De novembre 2008 à octobre 2009, la croissance a été en faveur de la longueur car les valeurs de b ont été significativement $<3$, correspondant ainsi à une croissance allomètrique négative. Ces déviations de croissance ont été si accentuées chez cette espèce qu'au cours de la saison des pluies de l'année 2008 (Mai-octobre), la croissance a été allométrique positive, mais est devenue allométrique négative à la même saison au cours de l'année suivante (2009). Arslan et al. (2004) expliquent ces déviations par le fait que b dépendrait des facteurs biotiques et abiotiques, surtout de la disponibilité des aliments et du type de l'habitat. Les valeurs du coefficient de variation b pour les mâles $(2,926)$, pour les femelles $(2,945)$, pour les individus à sexe indéterminé $(2,927)$ et pour la population totale $(2,980)$ dans la présente analyse sont très proches de 3,0; par conséquence Labeobarbus batesii de la plaine des Mbô aurait une croissance isométrique. Les résultats similaires ont été rapportés au Bangladesh chez Rhinomugil corsula (Mortuza et Rahman, 2006); au golfe de Tunis chez Trachurus trachurus et Trachurus mediterranus (Cherif et al., 2008) 
Le facteur de condition de Labeobarbus batesii a montré une variation mensuelle et a été en général > 1 . D'après Fulton (1902), $\mathrm{K} \geq 1$ exprime le «bien être » d'une population au cours des stades variés de son cycle de vie; alors que $\mathrm{K}<1$ signifie que le poisson n'est pas en embonpoint dans son biotope. Le «bien être » de la population de cette espèce se serait donc bien exprimé dans l'écosystème plaine inondable des Mbô au cours de cette période d'étude. Par ailleurs, le facteur de condition a oscillé entre les différentes tailles, c'est ainsi qu'on a enregistré des valeurs faibles et fortes aussi bien chez les juvéniles que chez les adultes. Ces résultats sont contraires de ceux rapportés par Tiogué et al. (non publié) chez la même espèce et dans la même zone au cours de 10 mois d'étude sur un échantillon de petite taille. Cette différence peut être expliquée par le fait que le facteur de condition varie non seulement en fonction de la disponibilité en aliment, mais aussi en fonction de la saison et des tailles des poissons, ainsi que de l'échantillon.

\section{Conclusion}

$\mathrm{Au}$ terme de cette étude sur la morphologie générale et les caractéristiques de croissance de Labeobarbus batesii dans la plaine inondable des Mbô, il ressort que c'est une espèce de grande taille. Elle a une croissance isométrique et un facteur de condition supérieur à 1 . Elle pourrait donc être exploitée comme espèce potentielle pour l'aquaculture, afin d'améliorer l'alimentation humaine. De plus, des études de cette nature sont importantes pour permettre de comprendre la biologie des carpes africaines qui est restée jusqu'ici un domaine vierge.

Toutefois, pour compléter cette étude, les caractéristiques reproductives et les habitudes alimentaires de cette espèce devront être étudiées.

\section{REMERCIEMENTS}

Les auteurs remercient: le Dr Brummett Randy E. récemment à World Fish Center pour sa contribution à l'identification de cette espèce ; tous les pêcheurs de la plaine inondable des Mbô et plus particulièrement Mrs Assoua Ewoussoua Bertrand et Fotem David qui ont contribué à l'identification des sites de collectes et à la capture des poissons.

\section{REFERENCES}

Arimoro OF, Orogun OE. 2008. Notes on the biology and ecology of Sudanonautes flowei (De Man, 1901; Crustacea: Brachyura: Potamoîdea: Potamonautidae) in River Ogbomwen, Southern Nigeria. Acta. Boil.Colomb., 13(1): 1-12.

Arslan M, Yidirim A, Bekta S. 2004. Lengthweight relationships of Brown Trout, Salmo trutta L., Inhabiting Kan Stream, Coruh Basin, North-eastern Turkey. Turkish J. Fisheries and Aquat. Sci., 4: 45-48.

Cherif M, Zarrad R, Gharbi H, Missaoui H, Jarboui O. 2008. Length-weight relationships for 11 fish species from the Gulf of Tunis (SW Mediterranean Sea, Tunisia). Pan-American Journal of Aquatic Sciences, 3(1): 1-5.

Chikou A. 2006. Etude de la démographie et de l'exploitation halieutique de six espèces de poissons-chats ( Teleostei, Siluriformes) dans le delta de l'Ouémé. Thèse de Doctorat, Université de Liège (XIII), p.370.

Coulibaly DN. 2008. Relation longueur-poids chez quatre espèces de poissons de la rivière Sourou au Burkina Faso. Int. J. Biol. Chem. Sci., 2(3): 331-338.

Fontaine P, Legendre M, Vandeputte M, Fostier A. 2009. Domestication de nouvelles espèces et développement durable de la pisciculture. Cah. Agric., 18(2-3): 119-124.

Fulton. 1902. Rate of growth of seas fishes. Sci. Invest. Fish. Div. Scot. Rept., 20: 1035-1039.

Lazard J. 2007. Aquaculture et espèces introduites: exemple de la domestication ex situ des tilapias. Doi : 10.1684/agr.2007 .0085. Cah. Agric., 16: 123-124. 
Lazard J, Levêque C. 2009. Introduction et transferts d'espèces de poissons d'eau douce. Cah.. Agric., 18(2-3): 157-163.

Le Cren ED. 1951. The length-weight relationships and seasonal cycle in gonad weight and condition in perch (Perca fluviatilis). Journal of animal Ecology, 20(2): 201-219.

Legendre M, Albaret JJ.1991. Maximum observed length (MOL) as an indicator of growth rate in tropical fishes. Aquaculture, 94: 327-341.

Lévêque C, Paugy D. 1999. Les Poisons des Eaux Continentales Africaines: Diversité, Ecologie, Utilisation par l'Homme. Editions IRD: Paris; $521 \mathrm{p}$.

Montcho AS, Laleye P, Linsenmair KE. 2009. Length-length, length-weight relationships and condition factor of Nile perch, Lates niloticus (Linnaeus, 1762) in the Pendjari River, West Africa. Int. J. Biol. Chem. Sci., 3(3): 466-474.

Montchowui E, Kogbeto MJ, Lalèyè P. 2009. Weight-length relationships for commercial fish species caught in Lake Hlan in Benin ( West Africa). Int. J. Biol. Chem. Sci., 3(3): 612-616.

Mortuza GM, Rahman T. 2006. Lengthweight relationship, condition factor and sex-ratio of freshwater fish, Rhinomugil corsula (Hamilton) (Mugiliformes: Mugilidae) from Rajshahi, Bangladesh. $J$. Bio-Sci., 14: 139-141.
Offem OB, Samsons AY, Omoniyi TI. 2009. Length-weight Relationship, Condition factor and Sex Ratio of Forty six Important Fishes in a Tropical Flood River. Research Journal of Fisheries and Hydrobiology, 4(2): 65-72.

Olivry JC. 1986. Fleuves et Rivières du Cameroun. Coll. Monographies Hydrologiques. Ed. MESRES-ORSTOM: Paris; p.733.

Pauly D, Gayanilo FCJr. 1997. Abee: An alternative approach to estimating the parameters of length-weight relationship from length-frequency samples and their bulk weights. ICLARM, Manila, Philippines. The FAO-ICLARM Stock Assessment Tool (FiSAT). Reference Manual, FAO Comp. Inf. Ser./Fish. 8, $262 \mathrm{p}$.

Pouomogne V. 2008. Capture-based aquaculture of Clarias catfish: case study of the Santchou fishers in western Cameroon, A Lovatelli, PF Holthus (eds). Capture-based aquaculture. Global overview. FAO Fisheries Technical Paper. No. 508, Rome, 93-108.

Ricker WE.1975. Stock and recruitment. In Reading in Aquatic Ecology, Ford, Hazen (eds), p .397.

Stiassny MLJ, Teugels GG, Hopkins CD. 2007. Poissons d'eaux douces et saumâtres de basse Guinée, Ouest de l'Afrique Centrale (Vol. 1). In Faune et Flore Tropicales. IRD Editions, Tervureen, MRAC : Paris ; 805. 